\title{
COMMENTARY
}

\section{Methicillin-resistant Staphylococcus aureus carriage at ICU admission: to screen (rapidly) or not to screen?}

\author{
Jean-Christophe Lucet* \\ See related research by Wassenberg et al., http://ccforum.com/content/16/1/R22
}

\begin{abstract}
The study by Wassenberg and colleagues shows that rapid diagnostic testing, although not cost-saving, reduces the number of unnecessary isolation days at ICU admission. Here, the strengths and limitations and the usefulness of rapid diagnostic testing are discussed from the collective and individual perspectives.
\end{abstract}

Methicillin-resistant Staphylococcus aureus (MRSA) has received much attention over the past 30 years because of its increasing incidence and the morbidity and excess hospital stay length associated with MRSA infection. The current controversy about the effectiveness of control measures, notably screening, derives from the conflicting results of available studies, lack of well-designed controlled studies, and concomitant use of several interventions in various epidemiological situations.

The 'search and destroy' strategy has kept MRSA under control in several countries and produced improvements in countries of MRSA endemicity. The most robust data about this strategy originate from northern European countries, where MRSA still occurs sporadically in hospitals. Wassenberg and colleagues [1] report a multicenter study evaluating the impact of rapid diagnostic testing (RDT) using PCR at ICU admission, which they compared to conventional screening. RDT reduced the number of pre-emptive isolation days by $44 \%$ in patients at high-risk for MRSA colonization. Cost-savings per isolation day avoided by earlier discontinuation of contact precautions varied between $€ 122$ and $€ 136$. The same authors recently demonstrated that RDT reduced

*Correspondence: jean-christophe.lucet@bch.aphp.fr

Infection Control Unit, AP-HP, Bichat - Claude Bernard Hospital, and Univ Paris Diderot, Sorbonne Paris Cité, 46 rue Henri Huchard, 75877 Paris cedex 18, France the number of pre-emptive isolation days by $60 \%$ in general wards [2]. In this last study, costs per isolation day avoided varied between $€ 96$ and $€ 125$. The use of chromogenic agar, a strategy not tested in the present study, reduced the number of pre-emptive isolation days by $47 \%$ and was cost-saving [2].

The authors used enrichment broth and collected multiple samples to increase the likelihood of identifying MRSA carriers. Of the 163 patients tested, 14 were MRSA-positive by PCR (including the 5 patients with positive conventional cultures) and 8 had inconclusive PCR results. When conventional culture was used as the reference standard, the prevalence of MRSA was 3\%. The high rate of false-positive or inconclusive results mandates the use of conventional culture as the reference standard on which to base decisions. In addition, units or hospitals using decolonization need to perform regular tests of susceptibility to the decolonization measures, notably mupirocin.

In the study by Wassenberg and co-workers, the high sensitivity of RDT allowed the discontinuation of contact precautions in patients with negative PCR results at admission. In other studies, however, sensitivity was lower than 95\% [3]. Sensitivity below 95\% in ICUs with MRSA prevalences higher than the $3 \%$ observed by Wassenberg and colleagues would result in a lower negative predictive value, with a risk of false-negative PCR results in carriers that would jeopardize the effectiveness of control policies. The decrease in negative predictive value with increasing admission MRSA prevalence, together with the possible reduction in contact precaution compliance when the number of patients to be isolated is high, suggests that RDT may be most useful in units with a low MRSA prevalence at admission.

The median turnaround times (TATs) for the IDIMRSA BD GeneOhm and GeneXpert MRSA system RDT from the start of isolation to definitive test results were 25.2 and 21.1 hours, respectively. One participating ICU in the IDI-MRSA BD GeneOhm group was late in 
delivering swabs to the laboratory, which increased the median TAT. However, it is difficult to understand why the total median TAT was 21.1 hours in the GeneXpert group, given that the median time to swab arrival at the laboratory was 5.8 hours and the median time from arrival to results was 2.6 hours. The short median time to obtaining results in the laboratory in the GeneXpert group is ascribable to the use of disposable cartridges and of a fully automated system, which obviated the need for a specialized technician. This test can be performed immediately at arrival at the laboratory or at the point of care. However, the long median TAT in the GeneXpert group suggests that some hospitals had delays in specimen delivery to the laboratory and in test performance, probably during nights and weekends.

If specimens are sent rapidly to the laboratory, and if testing is performed 24 hours a day and 7 days a week, RDT may be useful for guiding decisions. For instance, at hospital admission high-risk patients may be placed under contact precautions in a waiting/triage area with single rooms. A negative RDT result then allows the transfer of the patient to unrestricted areas. Patients with positive RDT results may be placed in single rooms with contact precautions, pending the result of the conventional culture. As indicated by the comparison between conventional culture and RDT, this strategy may be feasible only in hospitals with a triage unit and a low MRSA prevalence at hospital admission. However, there is no evidence so far that RDT is more effective than conventional culture in decreasing the MRSA acquisition rate [4].

In addition to the collective objective of placing colonized patients under contact precautions to limit MRSA dissemination, the prevention of MRSA infection in individual MRSA carriers may benefit from the use of RDT. Evidence supporting this possibility is scarce in the ICU setting [5,6], although MRSA infection prevention has been demonstrated when RDT was used before clean surgery [7]. Another study, however, found no benefits in surgical patients [8].

Costs of RDT were high, from $€ 120$ to $140 €$ per isolation day avoided. These costs were partly explained by the sampling of several sites. Grouping all screening samples into a single test, either RDT or conventional culture, would reduce costs [9]. Obtaining a screening card may be useful for deciding which sites should be decolonized. However, decolonization is not performed in all hospitals or countries, because of the absence of incontrovertible evidence of efficacy in hospitals with high MRSA rates, the lack of national policies, and high failure rates in mupirocin-resistant MRSA [10] and in patients with infected sites in whom decolonization would require systemic antibiotic.

The study by Wassenberg and colleagues adds to the vast literature about screening for MRSA at ICU admission. From a collective perspective, rapid diagnostic testing may reduce the number of unnecessary isolation days, provided that the test is performed in real time and the technique is sensitive enough for guiding decision in settings with low MRSA prevalence. Its usefulness and cost-effectiveness may be lower in endemic situations, in which chromogenic agar is an interesting option.

\section{Abbreviations}

MRSA, methicillin-resistant Staphylococcus aureus; PCR, polymerase chain reaction; RDT, rapid diagnostic testing; TAT, turnaround time.

\section{Competing interests}

$J C L$ has received fees from $3 \mathrm{M}$ company.

Published: 23 March 2012

\section{References}

1. Wassenberg M, Kluytmans J, Erdkamp S, Bosboom R, Buiting A, van Elzakker E, Melchers W, Thijsen S, Troelstra A, Vandenbroucke-Grauls C, Visser C, Voss A, Wolffs P, Wulf M, van Zwet T, de Wit A, Bonten M: Costs and benefits of rapid screening of methicillin-resistant Staphylococcus aureus carriage in intensive care units: a prospective multicenter study. Crit Care 2012, 16:R22

2. Wassenberg MW, Kluytmans JA, Box AT, Bosboom RW, Buiting AG, van Elzakker EP, Melchers WJ, van Rijen MM, Thijsen SF, Troelstra A, Vandenbroucke-Grauls CM, Visser CE, Voss A, Wolffs PF, Wulf MW, van Zwet $A A$, de Wit GA, Bonten MJ: Rapid screening of methicillin-resistant Staphylococcus aureus using PCR and chromogenic agar: a prospective study to evaluate costs and effects. Clin Microbiol Infect 2010, 16:1754-1761.

3. Luteijn JM, Hubben GA, Pechlivanoglou P, Bonten MJ, Postma MJ: Diagnostic accuracy of culture-based and PCR-based detection tests for methicillinresistant Staphylococcus aureus: a meta-analysis. Clin Microbiol Infect 2011, 17:146-154.

4. Tacconelli E, De Angelis G, de Waure C, Cataldo MA, La Torre G, Cauda R: Rapid screening tests for meticillin-resistant Staphylococcus aureus at hospital admission: systematic review and meta-analysis. Lancet Infect Dis 2009, 9:546-554.

5. Camus C, Bellissant E, Sebille V, Perrotin D, Garo B, Legras A, Renault A, Le Corre P, Donnio PY, Gacouin A, Le Tulzo Y, Thomas R: Prevention of acquired infections in intubated patients with the combination of two decontamination regimens. Crit Care Med 2005, 33:307-314.

6. Muller A, Talon D, Potier A, Belle E, Cappelier G, Bertrand X: Use of intranasal mupirocin to prevent methicillin-resistant Staphylococcus aureus infection in intensive care units. Crit Care 2005, 9:R246-250.

7. Walsh EE, Greene L, Kirshner R: Sustained reduction in methicillin-resistant Staphylococcus aureus wound infections after cardiothoracic surgery. Arch Intern Med 2011, 171:68-73.

8. Harbarth S, Fankhauser C, Schrenzel J, Christenson J, Gervaz P, Bandiera-Clerc C, Renzi G, Vernaz N, Sax H, Pittet D: Universal screening for methicillinresistant Staphylococcus aureus at hospital admission and nosocomial infection in surgical patients. JAMA 2008, 299:1149-1157.

9. Wassenberg MW, Kluytmans JA, Bosboom RW, Buiting AG, van Elzakker EP, Melchers WJ, Thijsen SF, Troelstra A, Vandenbroucke-Grauls CM, Visser CE, Voss A, Wolffs PF, Wulf MW, van Zwet AA, de Wit GA, Bonten MJ: Rapid diagnostic testing of methicillin-resistant Staphylococcus aureus carriage at different anatomical sites: costs and benefits of less extensive screening regimens. Clin Microbiol Infect 2011, 17:1704-1710.

10. Lee AS, Macedo-Vinas M, François P, Renzi G, Schrenzel J, Vernaz N, Pittet $D$, Harbarth S: Impact of combined low-level mupirocin and genotypic chlorhexidine resistance on persistent methicillin-resistant Staphylococcus aureus carriage after decolonization therapy: a casecontrol study. Clin Infect Dis 2011, 52:1422-1430.

\section{doi:10.1186/cc11222}

Cite this article as: Lucet JC: Methicillin-resistant Staphylococcus aureus carriage at ICU admission: to screen (rapidly) or not to screen? Critical Care 2012, 16:120 